\title{
The use of ketamine in the management of refractory cancer pain in a palliative care unit
}

\author{
Ka Wai Alice Cheung, Po Chung Chan, Sing Hung Lo \\ Department of Clinical Oncology, Tuen Mun Hospital, New Territories West Cluster, Hospital Authority, Hong Kong, China \\ Contributions: (I) Conception and design: All authors; (II) Administrative support: None; (III) Provision of study materials or patients: KW Cheung, \\ SH Lo; (IV) Collection and assembly of data: KW Cheung; (V) Data analysis and interpretation: KW Cheung, PC Chan; (VI) Manuscript writing: \\ All authors; (VII) Final approval of manuscript: All authors. \\ Correspondence to: Ka Wai Alice Cheung. Department of Clinical Oncology, Tuen Mun Hospital, 23 Tsing Chung Koon Road, Tuen Mun, Hong \\ Kong, China. Email: ckw045@ha.org.hk.
}

\begin{abstract}
Backgroundk Ketamine has been used as an adjuvant to opioid therapy for the management of refractory cancer pain but the current evidence is insufficient to draw any conclusions regarding its efficacy. We aimed to assess the response to ketamine in patients with refractory cancer pain treated in an oncology palliative care unit.

Methods: Patients with refractory cancer pain despite opioid dose escalation were selected for a trial of parenteral ketamine infusion according to a local protocol. The medical records of those patients treated between January 2004 and December 2018 were retrospectively reviewed. The primary endpoint of the study was a favorable response to ketamine, defined as a reduction in regular opioid dose with no increase in pain intensity or a reduction in pain intensity by $\geq 2$ points on the numerical rating scale (NRS) with a stable regular opioid dose. The secondary endpoint was adverse events associated with ketamine.
\end{abstract}

Results: Among the 70 patients, mean pain score on NRS improved from 7.0 to 4.0 after ketamine $(\mathrm{P}<0.001)$. Forty-nine patients had a reduction of pain score by $\geq 2$ points on NRS, 33 had $\geq 50 \%$ reduction in pain intensity. The median decrease in regular opioid dose was $25.5 \%$, and the mean difference was $-133.2 \mathrm{mg}(\mathrm{P}=0.002)$. A favorable response to ketamine was observed in 52 patients $(74.3 \%)$. The use of more than one coanalgesic (odds ratio 3.451; 95\% CI: 1.087-10.960; $\mathrm{P}=0.036$ ) was associated with a favorable response to ketamine on multivariate analysis. Adverse events were mostly mild, with the commonest being drowsiness (45.7\%), hypertension (34.3\%) and nightmares (25.7\%). Only five and three patients required temporary suspension and early termination of ketamine infusion respectively.

Conclusions: These data demonstrated the efficacy and safety of ketamine in a population of patients with refractory cancer pain. The use of more than one coanalgesic was associated with a favorable response to ketamine. Further large and multicentered studies are warranted to confirm these data.

Keywords: Cancer pain; intractable pain; ketamine; neoplasms; palliative care

Submitted Apr 14, 2019. Accepted for publication Sep 03, 2019.

doi: 10.21037/apm.2019.09.09

View this article at: http://dx.doi.org/10.21037/apm.2019.09.09

\section{Introduction}

Pain is commonly encountered in cancer patients, with an overall prevalence of $50.7 \%$ in all cancer stages (1). Among these patients, at least $20 \%$ to $40 \%$ of the pain is not adequately relieved by standard treatments $(2,3)$. In the literature, refractory cancer pain has been defined as pain related to cancer or its treatment, that persisted over time, generally at least three months, despite an adequate trial of analgesic therapies, including opioids, coanalgesics and non-pharmacological approaches $(4,5)$.

Ketamine has been used as a general anesthetic agent 
for over 30 years (6). It has also been utilized off-label, at subanesthetic doses, in the management of cancer pain, usually in conjunction with opioids. A usual indication for ketamine is in the setting of refractory cancer pain. In the 1980s, ketamine was discovered to have N-methyl-Daspartate (NMDA) receptor antagonist properties $(7,8)$. NMDA receptors are closely involved in the development of central sensitization of dorsal horn neurons, which transmit pain signals (9). Central sensitization occurs with ongoing nociceptive input from inflammation or injury, leading to persistent and escalating pain, hyperalgesia, allodynia and relative non-responsiveness to opioids in chronic pain. Binding of ketamine to these receptors could potentially reverse these phenomena (10).

Animal studies have demonstrated an antihyperalgesic effect of NMDA receptor blockers in the rat models of chronic pain (11), while a number of human volunteer studies, observational studies and small clinical trials have indicated that subanesthetic doses of ketamine alleviate various chronic and neuropathic pain syndromes (6). However, in the multisite, randomized controlled trial (RCT) published in 2012 (12), no additional clinical benefit was found for ketamine in the management of chronic uncontrolled pain related to cancer or its treatment, while causing significant toxicities. The use of ketamine has been debated in terms of scientific evidence, the 2017 Cochrane review concluded that the current evidence is insufficient to assess the benefits and harms of ketamine as an adjuvant to opioids for relief of refractory cancer pain (13). The aim of this study was to assess the response to ketamine in patients with refractory cancer pain treated in an oncology palliative care unit.

\section{Methods}

\section{Ketamine infusion protocol for refractory cancer pain}

Ketamine has been utilized in the management of refractory cancer pain in our palliative care unit. A local protocol has been established in 2003. Patients having nociceptive or neuropathic cancer pain with unsatisfactory control despite standard pain therapy, specifically regular opioid use with an oral morphine equivalent (OME) dose of $\geq 300$ milligrams (mg) daily for $>24$ hours, will be considered for a trial of ketamine infusion. Other criteria include relative nonresponsiveness to opioids, opioid tolerance associated with undesirable side effects and opioid-induced hyperalgesia, generally the features suggestive of central sensitization.
Cases need to be screened by palliative care specialists in the department before ketamine is commenced, to ensure appropriate case selection. Exclusion criteria include history of psychosis, severe labile hypertension, poorly controlled arrhythmia and hypersensitivity to ketamine.

Continuous subcutaneous ketamine [KetalarTM $50 \mathrm{mg} / \mathrm{milliliters} \mathrm{(mL),} \mathrm{Pfizer]} \mathrm{infusion} \mathrm{is} \mathrm{given} \mathrm{in} \mathrm{Oncology}$ wards, usually started at $100 \mathrm{mg}$ (diluted to $7.8 \mathrm{~mL}$ with normal saline) infused over 24 hours using a portable syringe driver, titrated daily according to the pain response and side effects. A maximum increment of $100 \mathrm{mg}$ of ketamine per day is allowed, normally up to a total dose of $300 \mathrm{mg}$ daily.

Pain intensity is to be recorded before ketamine administration, at least daily during ketamine infusion and upon discontinuation of ketamine infusion. Pain intensity is evaluated using numerical rating scale (NRS) on an 11-point Likert scale (from 0 to 10 , with 10 being worst pain imaginable); a verbal rating scale on a fourpoint categorical scale (from zero to three, signifying no, mild, moderate and severe pain respectively) would be used if the patient has difficulty in quantifying the pain on NRS. Blood pressure and pulse rate are measured by Dinamap ${ }^{\circledR}$ Vital Signs Monitor, normally at a frequency of four times daily during the infusion period. Psychomimetic side effects, local injection site reaction and other side effects of ketamine are assessed clinically, at least daily, with direct questioning about presence of hallucinations and nightmares. All administrations of analgesics and coanalgesics are documented in the hospital notes. Dose of regular opioids will be titrated during the ketamine treatment period, according to the pain response and side effects. It is suggested to reduce regular opioid dose daily by $20 \%$ of the previous dose, as long as the pain control is satisfactory. Ketamine is to be withdrawn slowly over two to three days subsequently.

\section{Study design and patients}

This was a retrospective cohort study. All consecutive patients who had been treated with parenteral ketamine for the management of refractory cancer pain from January 2004 to December 2018 in our palliative care unit were included. For patients who had received more than one episode of parenteral ketamine infusion, only the first episode would be analyzed, in order to avoid the confounding effect of repeated measures by repeatedly administering ketamine to some patients. Patients treated 
with oral ketamine were excluded.

\section{Data collection}

Data were collected from the date of commencement till the date of discontinuation of parenteral ketamine infusion. Pain assessment was regularly performed by attending Oncologists and nurses in all patients admitted to Oncology wards, regardless of study participation, and was prospectively collected using standardized pain assessment forms. All drug administrations were routinely documented. The pain assessment forms, drug administration records and other medical records were retrospectively reviewed. Demographic data and clinical information were collected. The data of opioid therapy and doses expressed as OME, and prescriptions of coanalgesics, upon initiation and termination of ketamine, were gathered. The morphine:methadone ratio adopted here was a dosedependent one: 4:1, 8:1 and 12:1 for daily morphine doses of 30-90, 91-300 and $>300 \mathrm{mg}$ respectively (14). Details about ketamine prescription, including the interruption of ketamine treatment or the occurrence of relevant adverse events, were examined. Baseline serum albumin levels, the doses of opioid therapy and coanalgesics at discharge were retrieved from the electronic medical records.

\section{Study outcomes}

The pain intensity and regular opioid dose before starting and upon stopping ketamine infusion were compared. The primary endpoint of the study was a favorable response to ketamine, which was defined as a reduction in regular opioid dose with no increase in pain intensity or a reduction in pain intensity by $\geq 2$ points on NRS (15) with a stable regular opioid dose. The secondary endpoint was adverse events associated with ketamine.

\section{Statistical analysis}

Descriptive analysis was performed with categorical data presented as counts and percentages. Continuous data were described using mean, median, minimum and maximum values, and interquartile range. For pain intensity scores, missing data were imputed from the last recorded assessment of pain score. Pain intensity scores and daily regular opioid dose before and after ketamine infusion were compared using paired-samples $t$-test. Categorical variables were compared by Pearson Chi-square test, or Fisher's exact test when the expected value in any of the cells in the contingency table was below five, while continuous variables were compared by independent-samples $t$-test and Mann Whitney U test. Multivariate analysis using binary logistic regression was applied to test the independent significance of different covariates, where variables trending toward significance on univariate analysis $(\mathrm{P}<0.10)$ were included. The variables were fitted into the model using forward selection. Statistical analysis was performed using International Business Machines Statistical Package for the Social Sciences $\left(\mathrm{SPSS}^{\circledR}\right.$ ) Software version 22. All P values were two-sided and $\mathrm{P}<0.05$ was considered statistically significant.

\section{Results}

Eighty-four palliative care cancer patients had been treated with parenteral ketamine infusion for control of refractory cancer pain between January 2004 and December 2018. Thirteen patients were excluded from the analysis as their medical records were unable to be retrieved. Another patient was excluded as she had been put on oral ketamine upon discontinuing parenteral ketamine infusion. Seventy patients were eligible for analysis.

\section{Demographic characteristics (Table 1)}

The median age of the cohort was 56.6 years, $68.6 \%$ were male. The commonest cancer diagnoses were lung, head and neck and lower gastrointestinal. Most of the patients had distant metastases, with $21.4 \%$ having $\geq 3$ metastatic sites. The median length of hospital stay was 33 days, 37 patients were successfully discharged after ketamine infusion.

The majority of the patients had an Eastern Cooperative Oncology Group (ECOG) performance status (PS) of 2 and 3 . The median serum albumin was $34 \mathrm{~g} / \mathrm{L}$.

\section{Baseline pain assessment}

At baseline before commencing ketamine infusion, pain assessment was performed for all patients (Table 2). All the pain was due to the underlying cancer; the commonest sites were chest, mixed and pelvis. Pain nature was mostly mixed, with neuropathic component present in $65.7 \%$. All patients had a pain intensity scoring $\geq 5$ points on NRS, mean pain score was 7.0, there were eight patients who scored the pain as the worst pain imaginable. 
Table 1 Demographic characteristics

\begin{tabular}{|c|c|}
\hline Characteristics & Number $(\mathrm{n}=70)$ \\
\hline \multicolumn{2}{|l|}{ Gender } \\
\hline Male & $48(68.6)$ \\
\hline Female & $22(31.4)$ \\
\hline Age at cancer diagnosis, years & $54.3[30.2-78.1]$ \\
\hline Age on admission, years & $56.6[30.5-86.2]$ \\
\hline \multicolumn{2}{|l|}{ Cancer diagnosis } \\
\hline Lung & $28(40.0)$ \\
\hline Head and neck & $9(12.9)$ \\
\hline Lower gastrointestinal & $7(10.0)$ \\
\hline Pancreaticobiliary & $6(8.6)$ \\
\hline Unknown primary & $4(5.7)$ \\
\hline Prostate & $3(4.3)$ \\
\hline Upper gastrointestinal & $3(4.3)$ \\
\hline Gynecological & $2(2.9)$ \\
\hline Melanoma & $2(2.9)$ \\
\hline Urinary tract & $2(2.9)$ \\
\hline Liver & $1(1.4)$ \\
\hline Sarcoma & $1(1.4)$ \\
\hline Spinal astrocytoma & $1(1.4)$ \\
\hline Thyroid & $1(1.4)$ \\
\hline \multicolumn{2}{|l|}{ Presence of distant metastases } \\
\hline $\mathrm{Nil}$ & $9(12.9)$ \\
\hline \multicolumn{2}{|l|}{ No. of metastatic sites } \\
\hline 1 site & $25(35.7)$ \\
\hline 2 sites & $21(30.0)$ \\
\hline 3 sites & $10(14.3)$ \\
\hline$>3$ sites & $5(7.1)$ \\
\hline \multicolumn{2}{|l|}{ Mode of discharge from ward } \\
\hline Discharged home & $37(52.9)$ \\
\hline Died during admission & $33(47.1)$ \\
\hline Length of hospital stay (days) & $33[8-164]$ \\
\hline \multicolumn{2}{|l|}{ ECOG PS } \\
\hline 1 & $2(2.9)$ \\
\hline 2 & $40(57.1)$ \\
\hline 3 & $28(40.0)$ \\
\hline Serum albumin (g/L) & 34 [17-42] \\
\hline
\end{tabular}

Data are $\mathrm{n}(\%)$ or median [range]. ECOG, Eastern Cooperative Oncology Group; PS, performance status.

\section{Baseline use of analgesics and coanalgesics}

All patients were on regular opioids. The majority (91.4\%) had morphine as the main opioid drug, while four patients had oxycodone and two had fentanyl. All patients except one were on methadone as adjuvant analgesic. The median regular opioid dose, expressed in OME, was $425 \mathrm{mg} /$ day (interquartile range, $380-538 \mathrm{mg} /$ day).

Coanalgesics were used in 66 patients (94.3\%), with 42 patients $(60 \%)$ taking $\geq 2$ coanalgesics. The most commonly prescribed coanalgesics included gamma-aminobutyric acid (GABA) analogues $(n=44,62.9 \%)$, antidepressants $(n=39$, $55.7 \%)$ and benzodiazepines ( $\mathrm{n}=30,42.9 \%)$.

\section{Ketamine prescription}

Ketamine was given via subcutaneous infusion in 69 patients and through intravenous route in the remaining one. The median starting and maximum doses were $100 \mathrm{mg} /$ day (range, 50-200 mg/day) and $200 \mathrm{mg} /$ day (range, $50-400 \mathrm{mg} /$ day) respectively. The median duration of ketamine treatment was 10 days (interquartile range, $6.2-15$ days). In the 53 patients $(75.7 \%)$ who had dose increment of ketamine after commencement of infusion, the median rate of dose increment to the maximum dose was $35.7 \mathrm{mg} /$ day (interquartile range, $25-50 \mathrm{mg} /$ day).

\section{Pain control}

Pain intensity, in terms of mean pain score on NRS, improved from 7.0 to 4.0 after ketamine $(\mathrm{P}<0.001)$. Fortynine patients had a reduction of pain score by $\geq 2$ points on NRS (Table 3); 44 patients had $\geq 30 \%$ reduction in pain intensity over baseline while $33 \mathrm{had} \geq 50 \%$ reduction.

\section{Changes in opioid dose}

The median regular opioid dose in OME reduced from 425 to $345 \mathrm{mg} /$ day, with the median change being $-25.5 \%$ and the mean difference $-133.2 \mathrm{mg}(\mathrm{P}=0.002)$. Forty-eight patients $(68.6 \%)$ had a reduction in daily regular opioid dose (Table 3), among these patients the median decrease in total regular opioid dose was $36.8 \%$ (interquartile range, 24-59.4\%).

In the 37 patients $(52.9 \%)$ who were successfully discharged home after ketamine administration, the median regular opioid dose in OME upon stopping ketamine and upon discharge were comparable, which were 345 and 
Table 2 Baseline pain assessment

\begin{tabular}{|c|c|}
\hline Pain assessment items & Number $(n=70)$ \\
\hline \multicolumn{2}{|l|}{ Pain site } \\
\hline Head and neck & $7(10.0)$ \\
\hline Chest & $16(22.9)$ \\
\hline Abdomen & $6(8.6)$ \\
\hline Pelvis & $12(17.1)$ \\
\hline Back & $8(11.4)$ \\
\hline Lower limb & $8(11.4)$ \\
\hline Mixed & $13(18.6)$ \\
\hline \multicolumn{2}{|l|}{ Pain intensity } \\
\hline NRS 0-4/10 & $0(0.0)$ \\
\hline NRS 5-6/10 & 27 (38.6) \\
\hline NRS $7-10 / 10$ & $43(61.4)$ \\
\hline \multicolumn{2}{|l|}{ Pain nature } \\
\hline Musculoskeletal & $13(18.6)$ \\
\hline Visceral & $11(15.7)$ \\
\hline Neuropathic & $8(11.4)$ \\
\hline \multicolumn{2}{|l|}{ Mixed } \\
\hline Musculoskeletal and neuropathic & $30(42.9)$ \\
\hline Visceral and neuropathic & $8(11.4)$ \\
\hline \multicolumn{2}{|l|}{ Cause of pain } \\
\hline Cancer & $70(100.0)$ \\
\hline Noncancer & $0(0.0)$ \\
\hline Mixed & $0(0.0)$ \\
\hline
\end{tabular}

Data are $\mathrm{n}(\%)$. NRS, numerical rating scale.

Table 3 Pain control outcomes after ketamine infusion

\begin{tabular}{lc}
\hline Outcomes & Number $(n=70)$ \\
\hline Reduction of pain score $\geq 2$ points on NRS & $49(70.0)$ \\
Reduction in pain intensity $\geq 30 \%$ over baseline & $44(62.9)$ \\
Reduction in pain intensity $\geq 50 \%$ over baseline & $33(47.1)$ \\
Reduction in daily regular opioid dose & $48(68.6)$ \\
\hline
\end{tabular}

Data are $\mathrm{n}(\%)$. NRS, numerical rating scale.
$350 \mathrm{mg}$ respectively $(\mathrm{P}=0.336)$.

\section{Favorable response to ketamine}

Favorable response to ketamine was observed in 52 patients (74.3\%), with 48 patients $(68.6 \%)$ having a reduction in regular opioid dose with no increase in pain intensity and four patients $(5.7 \%)$ having a reduction in pain intensity while on a stable opioid dose. In the favorable response cohort, the mean pain score dropped from 7.1 to 3.4 on NRS $(\mathrm{P}<0.001)$ after ketamine; whereas in the unfavorable response cohort, the mean pain score was 6.9 before ketamine and 5.8 afterwards $(\mathrm{P}=0.126)$.

Table 4 showed the different characteristics of the patients in the favorable and unfavorable response cohorts. On univariate analysis, the factors that were associated with response to ketamine included the mode of discharge from ward $(\mathrm{P}=0.016)$, the maximum dose of ketamine infused $(\mathrm{P}=0.031)$ and the use of more than one coanalgesic $(\mathrm{P}=0.050)$, while serum albumin $>35 \mathrm{~g} / \mathrm{L}$ also had a trend towards statistical significance $(\mathrm{P}=0.069)$. Among these factors, the use of more than one coanalgesic (odds ratio 3.451; 95\% CI: $1.087-10.960 ; \mathrm{P}=0.036$ ) remained significantly associated with a favorable response to ketamine on multivariate analysis.

\section{Other interventions within four weeks of ketamine therapy}

There were 46 patients $(65.7 \%)$ who had received other interventions for pain control within four weeks of ketamine therapy. Fifteen patients $(21.4 \%)$ had received systemic anticancer treatment, 32 (45.7\%) had been given palliative radiotherapy to the pain site(s) and $10(14.3 \%)$ had undergone interventional procedure for pain control. Six $(8.6 \%)$ and three $(4.3 \%)$ patients had received two and three modalities of intervention respectively. The percentage of patients having received any other interventions for pain control was similar in the favorable and unfavorable response cohorts $(\mathrm{P}=0.774)$ (Table 4).

\section{Adverse events}

The commonest adverse event (Table 5) encountered 
Table 4 Comparison between favorable and unfavorable response cohorts

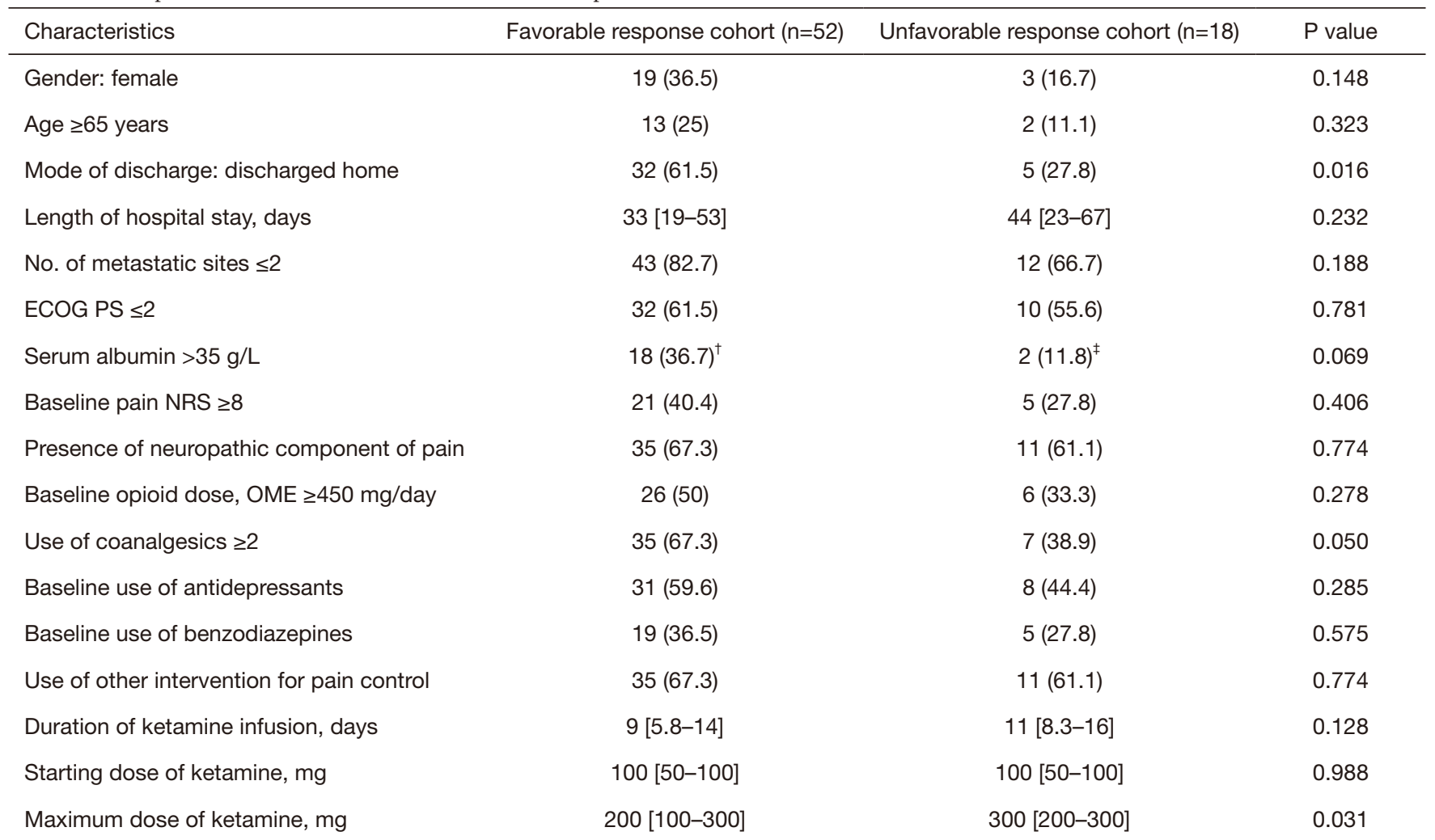

Data are $n(\%)$ or median [interquartile range]. ${ }^{\dagger}, n=49$, as 3 patients did not have serum albumin level checked within four weeks before starting ketamine. ${ }^{\ddagger}, \mathrm{n}=17$, as 1 patient did not have serum albumin level checked within four weeks before starting ketamine. ECOG, Eastern Cooperative Oncology Group; PS, performance status; NRS, numerical rating scale; OME, oral morphine equivalent; mg, milligrams.

during ketamine infusion was drowsiness, followed by hypertension, nightmares, local injection site reaction and confusion. There were no records of hemodynamic changes or arrhythmias attributable to ketamine administration.

Most of the adverse events were mild in severity. However, there were nine patients $(12.9 \%)$ who had a period of temporary suspension of ketamine, five of them (7.1\%) were attributed to side effects associated with ketamine: hypertension $(\mathrm{n}=2)$, drowsiness $(\mathrm{n}=2)$ and confusion $(\mathrm{n}=1)$. Other reasons included patient's refusal $(n=1)$, other intercurrent problem unrelated to ketamine $(\mathrm{n}=1)$, facilitation of home leave arrangement $(\mathrm{n}=1)$ and unknown reason $(\mathrm{n}=1)$.

Eight patients (11.4\%) required early termination of ketamine, before any intended clinical outcome could be achieved. Only three of them $(4.3 \%)$ were attributed to side effects associated with ketamine, specifically confusion $(n=2)$ and drowsiness $(n=1)$. Reasons in the other five cases were patient's refusal $(n=3)$ and other intercurrent problems (sepsis, $\mathrm{n}=1$; opioid overdose, $\mathrm{n}=1$ ).

\section{Discussion}

This retrospective review of 70 patients with refractory cancer pain who had received parenteral ketamine treatment in an oncology palliative care unit provided relevant information from a clinical perspective. According to a local protocol, strict selection criteria were adopted to test the efficacy of ketamine in cases with very difficult pain control. The study has found a favorable response to ketamine in pain control in $74.3 \%$ of patients. The use of more than one coanalgesic was associated with a favorable response to ketamine on multivariate analysis. Adverse events were mostly mild.

Ketamine has been utilized as an analgesic in a wide range of clinical settings. The efficacy of ketamine in the perioperative pain setting is well-established in a number of meta-analyses (16), while the evidence is lacking for most other indications. Although it may not be appropriate to directly extrapolate the data from acute perioperative pain to chronic cancer pain, it is in fact challenging, or even 
Table 5 Adverse events that occurred during ketamine infusion

\begin{tabular}{lc}
\hline Adverse events & Number $(\mathrm{n}=70)$ \\
\hline Central nervous system & $32(45.7)$ \\
Drowsiness & $18(25.7)$ \\
Nightmares & $10(14.3)$ \\
Confusion & $9(12.9)$ \\
Hallucinations & $5(7.1)$ \\
Vivid imagery & $1(1.4)$ \\
Irrational behavior & \\
Cardiovascular & $24(34.3)$ \\
Hypertension & $1(1.4)$ \\
Hypotension & \\
Gastrointestinal & $2(2.9)$ \\
Nausea & $2(2.9)$ \\
Vomiting & $11(15.7)$ \\
Local injection site & \\
Pain & \\
Local injection site reaction & \\
Ophthalmic & \\
Nystagmus & $1(10.0)$ \\
\hline
\end{tabular}

Data are $\mathrm{n}(\%)$.

considered to be inappropriate and infeasible by some, to conduct RCTs in patients with life-limiting disease, not to mention the complex pathophysiology of cancer pain. There exist ample preclinical evidence and animal studies which suggest NMDA receptor is essential for central sensitization $(11,17)$ and the antihyperalgesic effect through blocking these receptors has been demonstrated in many case reports and small uncontrolled studies (18-23). A longitudinal cohort study focussing in the pediatric population with various pain types showed that ketamine was specifically efficacious in malignancy-associated pain (24).

The Cochrane Database of Systematic Reviews had issued an update in 2017, adding the well-performed, blinded, adequately-powered RCT by Hardy et al. (12) to the last review in 2012 (25). It concluded that current evidence is insufficient to draw any conclusions regarding the efficacy of ketamine in cancer pain (13). The authors found that it was not possible to perform a quantitative meta-analysis of the three included RCTs due to the lack of extractable data, general heterogeneity of the data and the small number of participants in two of the trials $(19,26)$. The study by Hardy et al. (12) was a negative one, with a strong placebo response rate of $27 \%$ and a low number needed to harm of six. A specific ketamine treatment regimen was adopted in the trial一a dose-escalating regimen to $500 \mathrm{mg}$ /day given subcutaneously over five days, of which the extent of clinical utilization, the short period of exposure to the trial agent before it was deemed failure, and the safety of the dose increment rate have been thrown into doubt by some other experts in the field $(13,27,28)$. Another potential limitation of the trial was the heterogeneity of the study population. Researchers thus argue that the negative results should not discard the potential role of ketamine in all cancer patients with difficult pain control. In fact, two systematic reviews affirmed the role of ketamine in refractory cancer pain $(29,30)$, while Salas et al. found that specific populations could be "good responders" to ketamine (18).

Despite the lack of conclusive clinical evidence concerning its efficacy, clinicians continue to use ketamine as an adjuvant to opioids for chronic pain management (31). Many remained convinced of the benefit of the drug from their own positive clinical experience, some did not regard one negative RCT to be sufficient to induce a change in practice, others perceived that ketamine might have a role in specific patients. Practically, in the setting of limited effective pharmacological options to deal with refractory pain, one might be left with no alternative other than adopting less-studied strategies such as ketamine infusions. After all, further clinical evidence to support the use of ketamine has been long awaited. Similar to the situation of chronic noncancer pain, further studies are warranted to conclude the effect of ketamine, to define the target populations, as well as to determine the optimal dosage and regime of ketamine administration (32).

This study has demonstrated that $74.3 \%$ of the cohort receiving ketamine had a favorable response, either in terms of reduction in requirement of regular opioids or reduction in pain intensity. Average pain score dropped from 7 to 4 on NRS, with $70 \%$ of patients having a reduction of pain score by $\geq 2$ points on NRS, $62.9 \%$ having $\geq 30 \%$ reduction in pain intensity over baseline and $47.1 \%$ having $\geq 50 \%$ reduction. These are very appealing figures, given ketamine was applied to a population with difficult pain control.

We reported the outcomes in pain control in terms of change in pain score on NRS and percent pain relief. NRS is a standard instrument in chronic pain assessments, 
however, it does not contain any intrinsic meaning, patients may interpret measurement scales very differently when reporting pain, thus making the baseline scores widely variable between individuals (33). By calculating the raw change or percent change, the measures of improvement are adjusted for the individual's baseline. In 2001, Farrar et al. (15) found that, on average, a reduction of approximately two points or a reduction of approximately $30 \%$ in pain score on NRS represented a clinically important improvement. In recent years, researchers are getting more in favor of reporting the number or proportion of patients who have a clinically important decrease in pain, rather than using average pain scores or average change in pain scores. The Cochrane Pain, Palliative and Supportive Care Review Group (34) and the Initiative of Methods, Measurement, and Pain Assessment in Clinical Trials (IMMPACT) (35) have defined moderately important benefit and substantially important benefit as $\geq 30 \%$ reduction and $\geq 50 \%$ reduction in pain intensity over baseline. Pain reduction of $50 \%$ is used as the cutoff as this is regarded as a useful outcome according to patients (36), and is proven to be associated with major improvements in function, sleep, fatigue, depression, quality of life and work ability (37-40).

Our results were comparable to other published studies. Mercadante et al. (41) reported the results of a retrospective chart review in 2018 , consisting of 44 patients in total. Among them, $54.5 \%$ had $\geq 50 \%$ decrease in pain intensity and $75 \%$ had $\geq 30 \%$ decrease. There was a slight, statistically insignificant increase in opioid dose after ketamine as compared to baseline. The proportion of patients having $\geq 50 \%$ and $\geq 30 \%$ pain reduction was slightly lower in our study, $47.1 \%$ and $62.9 \%$ respectively, likely related to the statistically significant reduction in regular opioid dose after ketamine-mean reduction being $133.2 \mathrm{mg}(\mathrm{P}=0.002)$. Fitzgibbon et al. (42) has reported a better response rate in a retrospective audit in 2005, where 15 out of 16 patients $(93.8 \%)$ had a reduction in pain score of $\geq 4$ points on NRS, while the median opioid dose reduction was similar to us-25\% in Fitzgibbon's study and $25.5 \%$ in this study. On the other hand, the RCT by Salas et al. (18) found that 4 out of 11 patients receiving ketamine (36.4\%) had a reduction of $\geq 3$ points on visual analogue scale. Different definitions of clinically important pain control outcomes have been utilized in various studies, making direct comparison difficult. Nevertheless, the response rate to ketamine apparently varies widely across studies, and was even found to be negative in Hardy's
RCT (12). This may be related to the different criteria used for case selection: refractory pain was defined as pain with an intensity of $\geq 3$ points on an 11-point Likert scale despite ongoing treatment with opioids and coanalgesics, which has been criticized for not representing the clinical pattern commonly reserved for ketamine use.

It is also demonstrated here that ketamine administration offered a significant reduction in regular opioid dose, with the median regular opioid dose in OME reducing from 425 to $345 \mathrm{mg} /$ day. This is perceived to be a favorable outcome, as high and ever-escalating opioid dose would result in hyperalgesia and undesirable side effects.

In the literature, it has long been postulated that ketamine is useful in some, but not all, patients. There is so far no concrete conclusion as to what factors are associated with a favorable response to ketamine. Among children, adolescents and young adults, Sheehy et al. had reported that the effect of ketamine on pain scores varied according to clinical diagnosis, infusion duration and pain location (24). Mercadante et al. (41) had found no association between pain response and age, gender or pain mechanism, while Hardy et al. (12) stated that pain type was not a statistically significant predictor of response. In our cohort, $60 \%$ of patients were using more than one coanalgesic. This is common in patients who have poor response to opioids, usually involving pain with a neuropathic component. With this being the only factor that remained statistically significant in multivariate analysis, it implies that ketamine works surprisingly better in uncontrolled neuropathic pain, suggesting that pain which responds poorly to conventional analgesics, including coanalgesics, may be a selection criterion that warrants a trial of ketamine.

Ketamine was generally tolerable in our patients, in line with the findings by Mercadante et al. (41) and Sheehy et al. (24). Although adverse events were observed quite commonly, most of them were mild, except in $7.1 \%$ and $4.3 \%$ where temporary suspension and early termination of ketamine were indicated respectively. As compared to Hardy's RCT (12) where toxicities had been emphasized, a slower rate of dose increment of ketamine was adopted in our protocol, which may be associated with fewer adverse events (43).

\section{Limitations}

The principal limitation of this study is its retrospective nature, where data were retrieved from the charts. In our unit, attending Oncologists and nurses are required 
to perform regular, at least daily, pain assessments for all patients in Oncology wards using standardized pain assessment forms. It is also obligatory to document all drug administrations. These routines have helped to assure a reasonable quality of the data obtained from the charts.

Another concern is the potential of observer bias, which might affect the subjective assessment of pain, but probably has a lesser effect on the other outcome of our study-regular opioid dose. Here, the regular opioid dose was shown to have a significant reduction after the use of ketamine, signifying the success of ketamine as an adjunct analgesic, specifically stressing on the role of ketamine in patients with features of central sensitization when further escalation of opioids is ineffective in improving pain control but only bringing about side effects.

The absence of a placebo control is yet another limitation of this study, especially a high placebo response rate has been reported in previous studies on neuropathic pain (44-46) and up to $27 \%$ in Hardy's RCT (12). Possible explanations for placebo responses of this order of magnitude have been suggested: regression of pain intensity to the mean, patient's expectation of benefit, and the extra care and attention given to patients participating in trials (47). It was observed in our cohort that the regular opioid dose upon discharge was not statistically different from that upon stopping ketamine, meaning those patients were discharged with a statistically and clinically significant reduction of regular opioid dose over baseline. Ketamine infusion normally had already been stopped for a period of time when the patient was discharged; by that time any placebo effect associated with patient's expectation of benefit, the extra care and attention provided during ketamine infusion would have been washed out. Thus, the results are likely reflecting a genuine pain response.

In the study, apart from pain intensity, dose of opioid therapy was another major outcome. Only regular opioid doses, but not the dose and frequency of rescue medications for breakthrough pain, were taken into account. This might potentially lead to an underestimation of the opioid doses required and in turn an overoptimistic result concerning the efficacy of ketamine. However, as the need of breakthrough analgesia differs from day to day, this would lead to considerable difficulties in data analysis and interpretation. On the contrary, it would be unethical to limit or restrict the use of breakthrough analgesia.

This study had included patients on opioids other than morphine, compatible with the real-world situation.
However, in our practice, morphine is the dominant firstline opioid, while oxycodone and fentanyl are only used when morphine is not well-tolerated or is contraindicated. Thus, only a minority of patients in the cohort were not using morphine, making subgroup analysis examining the effect of the opioid type on the response of ketamine impossible.

With the departmental protocol in place, there is generally consistency regarding the indications for ketamine use and the administration regimen, but the timing of ketamine administration, and the titration of opioids and ketamine doses are left to the discretion of the attending oncologists. Thus it was not possible to conclude on any optimal dosage, regime, duration or timing of ketamine use in managing refractory chronic pain.

\section{Future directions}

There are still a number of unanswered questions regarding the use of ketamine in the management of refractory cancer pain. Researchers and palliative care specialists are eagerly awaiting some more conclusive clinical evidence. Studies examining the optimal regimen, route, dose and timing of ketamine are important. Whether ketamine is more effective in combination with specific opioids and in which specific populations are other issues of high interest.

Four core chronic pain outcome domains were suggested in IMMPACT recommendations 2008 (35), which included pain intensity presented as proportion of patients having substantial ( $\geq 50 \%)$ and moderately important $(\geq 30 \%)$ pain reduction, physical functioning, emotional functioning, and participant ratings of overall improvement. It would be desirable if future clinical trials can adopt these standardized outcome measures.

RCTs would be ideal in providing high-quality clinical evidence, but the strict protocols of RCTs often do not reflect the difficult conditions of a selected population, and make conduction in the palliative care settings demanding. Large and multicentered studies would be feasible alternatives. It would be worthwhile to consider international audit with priorly agreed-on response outcomes and toxicity monitoring, utilizing big data analytics through prospectively collected clinical data, and calling for all patients with refractory pain being prescribed ketamine to register. That would create a large patient population, facilitating more reliable and conclusive data analysis, allowing for planned subgroup analysis to be performed. 


\section{Conclusions}

This retrospective cohort study suggested that ketamine was useful, with acceptable safety, in a population of patients with refractory cancer pain. The use of more than one coanalgesic was associated with a favorable response to ketamine. Further large and multicentered studies are warranted to confirm these data.

\section{Acknowledgments}

Dr. Chi Sing Frank Wong, the Department Chief, for his general support.

Funding: None.

\section{Footnote}

Provenance and Peer Review: This article was commissioned by the Guest Editors (Rebecca Yeung and Tai Chung Lam) for the series "Integrating Palliative Medicine in Oncology Care: The Hong Kong Experience" published in Annals of Palliative Medicine. The article has undergone external peer review.

Conflicts of Interest: All authors have completed the ICMJE uniform disclosure form (available at http://dx.doi. org/10.21037/apm.2019.09.09). The series "Integrating Palliative Medicine in Oncology Care: The Hong Kong Experience" was commissioned by the editorial office without any funding or sponsorship. The authors have no other conflicts of interest to declare.

Ethical Statement: The authors are accountable for all aspects of the work in ensuring that questions related to the accuracy or integrity of any part of the work are appropriately investigated and resolved. The study was conducted in accordance with the Declaration of Helsinki (as revised in 2013). This is a retrospective analysis of charts of patients, informed consent was not considered to be necessary. The patients' personal data have been secured. The study has been approved by the local institutional review board, i.e., New Territories West Cluster Research Ethics Committee, Hospital Authority, Hong Kong (Ref No.: NTWC/REC/19025).

Open Access Statement: This is an Open Access article distributed in accordance with the Creative Commons Attribution-NonCommercial-NoDerivs 4.0 International
License (CC BY-NC-ND 4.0), which permits the noncommercial replication and distribution of the article with the strict proviso that no changes or edits are made and the original work is properly cited (including links to both the formal publication through the relevant DOI and the license). See: https://creativecommons.org/licenses/by-nc-nd/4.0/.

\section{References}

1. van den Beuken-van Everdingen MH, Hochstenbach LM, Joosten EA, et al. Update on prevalence of pain in patients with cancer: Systematic review and meta-analysis. J Pain Symptom Manage 2016;51:1070-90.e9

2. Twycross R, Harcourt J, Bergl S. A survey of pain in patients with advanced cancer. J Pain Symptom Manage 1996;12:273-82.

3. Weiss SC, Emanuel LL, Fairclough DL, et al. Understanding the experience of pain in terminally ill patients. Lancet 2001;357:1311-5.

4. Afsharimani B, Kindl K, Good P, et al. Pharmacological options for the treatment of refractory cancer pain - what is the evidence? Support Care Cancer 2015;23:1473-81.

5. Currow DC, Spruyt O, Hardy J. Defining refractory pain in cancer for clinicians and researchers. J Palliat Med 2012;15:5-6.

6. Fisher K, Codere TJ, Hagen NA. Targeting the N-Methyl-D-Aspartate receptor for chronic pain management: preclinical animal studies, recent clinical experience and future research directions. J Pain Symptom Manage 2000;20:358-73.

7. Anis NA, Berry SC, Burton NR, et al. The dissociative anesthetics, ketamine and phencyclidine, selectively reduce excitation of central mammalian neurons by $\mathrm{N}$-methylaspartate. Br J Pharmacol 1983;79:565-75.

8. Thomson AM, West DC, Lodge D. An N-methyl-aspartate receptor-mediated synapse in rat cerebral cortex: a site of action of ketamine? Nature 1985;313:479-481.

9. Richens A. The basis of the treatment of epilepsy: neuropharmacology. In: Dam M. editor. A practical approach to epilepsy. Oxford: Pergamon Press, 1991:75-85.

10. Quibell R, Prommer EE, Mihalyo M, et al. Ketamine. J Pain Symptom Manage 2011;41:640-9.

11. Swartjes M, Morariu A, Niesters M, et al. Nonselective and NR2B-selective N-methyl-D-aspartic acid receptor antagonists produce antinociception and long-term relief of allodynia in acute and neuropathic pain. Anesthesiology 2011;115:165-74. 
12. Hardy J, Quinn S, Fazekas B, et al. Randomized, doubleblind, placebo-controlled study to assess the efficacy and toxicity of subcutaneous ketamine in the management of cancer pain. J Clin Oncol 2012;30:3611-7.

13. Bell RF, Eccleston C, Kalso EA. Ketamine as an adjuvant to opioids for cancer pain. Cochrane Database Syst Rev 2017;6:CD003351.

14. Mercadante S. Opioid rotation for cancer pain: rationale and clinical aspects. Cancer 1999;86:1856-66.

15. Farrar JT, Young JP Jr, LaMoreaux L, et al. Clinical importance of changes in chronic pain intensity measured on an 11-point numerical pain rating scale. Pain 2001;94:149-58.

16. Jonkman K, Dahan A, van de Donk T, et al. Ketamine for pain. F1000Res 2017;6:1711.

17. Ji Y, Traub RJ. Spinal NMDA receptors contribute to neuronal processing of acute noxious and nonnoxious colorectal stimulation in the rat. J Neurophysiol 2001;86:1783-91.

18. Salas S, Frasca M, Planchet-Barraud B, et al. Ketamine analgesic effect by continuous intravenous infusion in refractory cancer pain: considerations about the clinical research in palliative care. J Palliat Med 2012;15:287-93.

19. Mercadante S, Arcuri E, Tirelli W, et al. Analgesic effect of intravenous ketamine in cancer patients on morphine therapy: a randomized, controlled, doubleblind, crossover, double-dose study. J Pain Symptom Manage 2000;20:246-52.

20. Jackson K, Ashby M, Martin P, et al. "Burst" ketamine for refractory cancer pain: an open-label audit of 39 patients. J Pain Symptom Manage 2001;22:834-42.

21. Soto E, Stewart DR, Mannes AJ, et al. Oral ketamine in the palliative care setting: a review of the literature and case report of a patient with neurofibromatosis type 1 and glomus tumor-associated complex regional pain syndrome. Am J Hosp Palliat Care 2012;29:308-17.

22. Lauretti GR, Lima IC, Reis MP, et al. Oral ketamine and transdermal nitroglycerin as analgesic adjuvants to oral morphine therapy for cancer pain management. Anesthesiology 1999;90:1528-33.

23. Bell R, Eccleston C, Kalso EA. Ketamine as an adjuvant to opioids for cancer pain. Cochrane Database Syst Rev 2003;(1):CD003351.

24. Sheehy KA, Lippold C, Rice AL, et al. Subanesthetic ketamine for pain management in hospitalized children, adolescents, and young adults: a single-center cohort study. J Pain Res 2017;10:787-795.

25. Bell RF, Eccleston C, Kalso EA. Ketamine as an adjuvant to opioids for cancer pain. Cochrane Database Syst Rev 2012;11:CD003351.

26. Yang CY, Wong CS, Chang JY. Intrathecal ketamine reduces morphine requirements in patients with terminal cancer pain. Can J Anesth 1996;43:379-83.

27. Jackson K, Franco M, William L, et al. Ketamine and cancer pain: the reports of my death have been greatly exaggerated. J Clin Oncol 2013;31:1373-4.

28. Bell RF, Jaksch W, Kalso EA. Interpreting the evidence: reply to Spruyt et al. J Pain Symptom Manage 2014;47:e2-4.

29. Bredlau AL, Thakur R, Korones DN, et al. Ketamine for pain in adults and children with cancer: a systematic review and synthesis of the literature. Pain Med 2013;14:1505-17.

30. van den Beuken-van Everdingen MH, de Graeff A, Jongen $\mathrm{JL}$, et al, the national guideline working group "Diagnosis treatment of cancer pain". Pharmacological treatment of pain in cancer patients: the role of adjuvant analgesics, a systematic review. Pain Pract 2017;17:409-19.

31. Hardy JR, Spruyt O, Quinn SJ, et al. Implementing practice change in chronic cancer pain management: clinician response to a phase III study of ketamine. Intern Med J 2014;44:586-91.

32. Michelet D, Brasher C, Horlin AL, et al. Ketamine for chronic non-cancer pain: A meta-analysis and trial sequential analysis of randomized controlled trials. Eur J Pain 2018;22:632-46.

33. Turk DC, Rudy TE, Sorkin BA. Neglected topics in chronic pain treatment outcome studies: determination of success. Pain 1993;53:3-16.

34. Pain, Palliative and Supportive Care Group. Authoring or assessing a Cochrane Protocol, Review, or Review Update for the PaPaS Review Group (AUREF). Available online: papas.cochrane.org/papas-documents

35. Dworkin RH, Turk DC, Wyrwich KW, et al. Interpreting the clinical importance of treatment outcomes in chronic pain clinical trials: IMMPACT recommendations. J Pain 2008;9:105-21.

36. O'Brien EM, Staud RM, Hassinger AD, et al. Patientcentered perspective on treatment outcomes in chronic pain. Pain Med 2010;11:6-15.

37. Zelman DC, Dukes E, Brandenburg N, et al. Identification of cut-points for mild, moderate and severe pain due to diabetic peripheral neuropathy. Pain 2005;115:29-36.

38. Barthel HR, Peniston JH, Clark MB, et al. Correlation of pain relief with physical function in hand osteoarthritis: randomized controlled trial post hoc analysis. Arthritis Res Ther 2010;12:R7. 
39. Hoffman DL, Sadosky A, Dukes EM, et al. How do changes in pain severity levels correspond to changes in health status and function in patients with painful diabetic peripheral neuropathy? Pain 2010;149:194-201.

40. Moore RA, Straube S, Paine J, et al. Fibromyalgia: Moderate and substantial pain intensity reduction predicts improvement in other outcomes and substantial quality of life gain. Pain 2010;149:360-4.

41. Mercadante S, Caruselli A, Casuccio A. The use of ketamine in a palliative-supportive care unit: a retrospective analysis. Ann Palliat Med 2018;7:205-10.

42. Fitzgibbon EJ, Viola R. Parenteral ketamine as an analgesic adjuvant for severe pain: development and retrospective audit of a protocol for a palliative care unit. J Palliat Med 2005;8:49-57.

Cite this article as: Cheung KW, Chan PC, Lo SH. The use of ketamine in the management of refractory cancer pain in a palliative care unit. Ann Palliat Med 2020;9(6):4478-4489. doi: 10.21037/apm.2019.09.09
43. Okamoto Y, Tsuneto S, Tanimukai H, et al. Can gradual dose titration of ketamine for management of neuropathic pain prevent psychotomimetic effects in patients with advanced cancer? Am J Hosp Palliat Care 2013;30:450-4.

44. McQuay HJ, Moore RA. Antidepressants and chronic pain. BMJ 1997;314:763-4.

45. Turner JA, Deyo RA, Loeser JD, et al. The importance of placebo effects in pain treatment and research. JAMA 1994;271:1609-14.

46. Irizarry MC, Webb DJ, Ali Z, et al. Predictors of placebo response in pooled lamotrigine neuropathic pain clinical trials. Clin J Pain 2009;25:469-76.

47. Farrar JT. Advances in clinical research methodology for pain clinical trials. Nat Med 2010;16:1284-93. 